Rabaska

Revue d'ethnologie de l'Amérique française

\title{
Les dialogues du folklore : la correspondance Massicotte-Barbeau
}

\section{Diane Joly}

Volume 13, 2015

Présence de Marius Barbeau : l'invention du terrain en Amérique

française. Autour d’un legs centenaire (1914-2014)

URI : https://id.erudit.org/iderudit/1033758ar

DOI : https://doi.org/10.7202/1033758ar

Aller au sommaire du numéro

Éditeur(s)

Société québécoise d'ethnologie

ISSN

1703-7433 (imprimé)

1916-7350 (numérique)

Découvrir la revue

Citer cet article

Joly, D. (2015). Les dialogues du folklore : la correspondance

Massicotte-Barbeau. Rabaska, 13, 153-163. https://doi.org/10.7202/1033758ar d'utilisation que vous pouvez consulter en ligne.

https://apropos.erudit.org/fr/usagers/politique-dutilisation/ 


\section{Deuxième partie}

Promotion et mise EN VALEUR DU PATRIMoine

\section{Barbeau et ses alliés}

\section{Les dialogues du folklore : la correspondance Massicotte-Barbeau}

DiAne JoLY

Consultante en patrimoine, Montréal

J'ai fini un index que j'ai recommencé parce que je voulais le rendre uniforme. Je prends invariablement le premier ou les deux premiers vers comme titre. $J$ 'y ajoute parfois un mot typique du refrain. Je mets aussi le numéro du cahier, la page, le nom du chanteur, si la chanson vous a été envoyée,

à quelle date vous en avez accusé réception. Plus la liste des chansons qui lui ressemblent, etc. Maintenant, j'entreprends une classification ${ }^{1}$. Édouard-Zotique Massicotte, 1918

L'histoire de 1'ethnologie québécoise retient d'Édouard-Zotique Massicotte l'image d'un collaborateur de Marius Barbeau bienveillant et dévoué à la tâche de recueillir du folklore ${ }^{2}$. Pourtant, leurs échanges épistolaires montrent une relation nuancée où apparaissent des divergences et des conflits. L'étude de ces sources manuscrites renseigne sur leurs liens professionnel et personnel de l'intérieur, car les archives possèdent ce côté caché, secret, même

1. Les Archives de folklore et d'ethnologie de l'Université Laval (AfeuL), Fonds ÉdouardZotique Massicotte (F837), É-Z. Massicotte à M. Barbeau, 25 janvier 1918.

2. Luc Lacourcière met en lumière la contribution hâtive de Massicotte aux recherches sur le folklore. Sœur Denise Rodrigue va plus loin en étudiant ses méthodes d'analyse qu'elle confronte aux modèles qui lui sont contemporains. Bien que celle-ci perçoive le caractère novateur des recherches de Massicotte, dans l'ensemble, elle le situe en amont des chercheurs modernes, précurseurs certes, mais encore amateurs si on les compare à Marius Barbeau formé en anthropologie. C'est cette dernière perception qui tend à être répétée par les chercheurs subséquents. Luc Lacourcière, «É.-Z. Massicotte et son œuvre folklorique », Les Archives de folklore, Québec, Fides, vol. 3, 1948, p. 7-12 ; Denise Rodrigue, «La Civilisation canadienne-française retracée dans les écrits de Édouard-Zotique Massicotte», Sainte-Foy, Université Laval, 1968. 
inconscient. Elles apportent un éclairage nouveau sur leur personnalité et mettent en lumière une relation qui connait des hauts et des bas pour finalement se fixer selon un mode de respect mutuel. La correspondance entre ces folkloristes s'est échelonnée sur trois décennies, de 1917 à 1947. On en retrouve des traces dans une douzaine de fonds d'archives dont au Service des archives du Musée canadien de l'histoire à Gatineau, au Centre d'archives de Montréal et aux Archives de folklore et d'ethnologie de l'Université Laval.

En 1917, Édouard-Zotique Massicotte a cinquante ans; il est archiviste à la ville de Montréal et réputé pour ses méthodes de travail rigoureuses. Plus jeune, il entreprend des études dans plusieurs domaines sans se décider. Il est tour à tour homme de théâtre, poète, écrivain et journaliste. Éventuellement, il entreprend des études en droit. Admis au Barreau en 1895, il abandonne rapidement la pratique et retourne au journalisme. Historien aguerri et méticuleux, il possède une connaissance étendue de la culture canadienne-française et des dizaines de publications à son actif ${ }^{3}$. Pour sa part, Marius Barbeau a trente-quatre ans. Il est à l'emploi de la division de l'anthropologie du musée de la Commission de géologie du Canada depuis 1911. Plus jeune, il entreprend des études en droit. Cependant, il se détourne de ce projet lorsqu'on lui octroie la prestigieuse bourse Rhodes. Il part alors pour l'Angleterre et décide d'étudier l'anthropologie. À cette époque, il séjourne également en France où il assiste à des cours offerts à l'École des hautes études, à la Sorbonne et à 1 'École d'anthropologie de Paris ${ }^{4}$. Au moment de sa rencontre avec Massicotte, Marius Barbeau est en début de carrière avec quelques publications et des connaissances historiques assez limitées. Dans son milieu professionnel, il est plutôt désorganisé, lent à répondre à son courrier et il perd des documents.

En termes de parcours et d'expérience, Édouard-Zotique Massicotte et Marius Barbeau sont assez éloignés l'un de l'autre. Ils se distinguent aussi sur leur appréhension du folklore et leur volonté de le diffuser. Massicotte perçoit le folklore comme une archive orale pour ses recherches sur la culture populaire et l'histoire. Depuis 1883, il tente d'amener ses compatriotes à s'y intéresser en leur offrant des textes accessibles où la majorité de ses com-

3. Parmi ses principales études sur le folklore : Mœurs, coutumes et industries d'autrefois, Montréal, Librairie Beauchemin, «Bibliothèque canadienne, collection Dollard », 1913 ; Conteurs canadiens-français au XIX siècle, Montréal, Beauchemin, 1902, et Monographies de plantes canadiennes (croquis champêtres et calendrier de la flore), Montréal, Beauchemin, 1899; «Folklore canadien : Les remèdes d'autrefois ", La Revue populaire, vol. 2, n 3, mars 1909, p. 51-53 ; " Folklore canadien : Superstitions », Le Terroir, juin 1909, p. 216-225 et « La Ceinture fléchée », Bulletin des recherches historiques, vol. 12, $\mathrm{n}^{\circ}$ 6, mai 1907, p. 154-156. Sa contribution au folklore avant sa rencontre avec Marius Barbeau est relatée dans Diane Joly, « À l'avant-garde du folklore : Édouard-Zotique Massicotte 1882-1915 », Rabaska, revue d'ethnologie de l'Amérique française, vol. 11, 2013, p. 25-41.

4. Vanessa Ferey, «Charles-Marius Barbeau et l'étude des collections ethnographiques francoaméricaines d'Europe de l'Ouest (1931-1956) », Rabaska, revue d'ethnologie de l'Amérique française, vol. 12, 2014, p. 89. 
mentaires visent à faire avancer des idées, à développer la culture et à les éduquer. Cette approche intimiste est une partie intégrante de son style d'écriture. Elle lui sert à introduire son sujet et à intéresser son public qui participe en lui soumettant de l'information notamment sur les chansons populaires. Massicotte conserve cette approche après sa rencontre avec l'anthropologue.

Au moins jusqu'en 1930, Marius Barbeau perçoit le folklore comme un outil patriotique pouvant servir à renouveler la littérature et la musique canadienne ou un remède pour combattre la modernité. Il veut asseoir ses compétences et sa crédibilité au sein de publications scientifiques surtout la revue Journal of American Folklore. Pour lui, le folklore est un sujet d'intérêt qu'il réserve aux intellectuels canadiens-français et aux spécialistes ${ }^{5}$.

Les deux chercheurs se complètent bien et leur association permet à Massicotte de se lancer à fond dans l'aventure du folklore puisque l'anthropologue lui prête de l'équipement technique de collecte, l'introduit à de nouveaux sujets de recherche et méthodes d'écriture. Auprès de Massicotte, Barbeau a accès à ses compétences en édition, ses connaissances historiques et sa minutie. Leur relation professionnelle s'est construite par les recherches sur le terrain et les collaborations de publication. Ils ont aussi travaillé ensemble brièvement lors des soirées de folklore en 1919 et 1920.

\section{Premiers contacts en mode séduction}

Édouard-Zotique Massicotte et Marius Barbeau se sont d'abord rencontrés par échanges épistolaires au cours de l'été 1917 sans doute grâce à Victor Morin. Proche de Massicotte, ce dernier est président de la Société historique de Montréal et il collabore à la fondation d'une section canadienne de l'American Folklore Society. L'archiviste envoie à l'anthropologue un résumé de ses cueillettes effectuées avant 1900 avec une liste d'études publiées sur le sujet. Après lecture, Barbeau lui demande s'il veut bien éditer ses notes pour une parution spéciale de la revue Journal of American Folklore ${ }^{6}$. Barbeau partage aussi avec Victor Morin son étonnement : « Les envois de monsieur Massicotte ont été une forte surprise pour moi et ils ont une réelle valeur. Il a obtenu des matériaux dans des domaines encore inexplorés dans Québec.

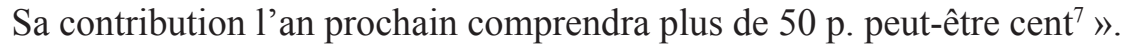

5. La carrière médiatique de Marius Barbeau auprès du grand public prend son envol en 1932 (Clarisse Cardin, " Bio-bibliographie de Marius Barbeau », Les Archives de folklore, " Hommage à Marius Barbeau », Montréal, Fides, vol. 2, 1947, p. 17-96).

6. É.-Z. Massicotte, « Croyances et dictons populaires des environs de Trois-Rivières (Canada) », Journal of American Folklore, vol. 32, n 123, 1919, p. 168-175; publiée à l'origine en 1909 dans La Revue populaire.

7. Centre des archives de l'Université de Montréal, Fonds Victor Morin (P56), M. Barbeau à V. Morin, 6 juillet 1917. 
Peu de temps après, Marius Barbeau envoie à Édouard-Zotique Massicotte le second volume de ses contes. On perçoit tout de suite la curiosité de celui-ci et ses connaissances sur le sujet lorsqu'il écrit : « Il y a un couple, au moins, de ces contes qui ont été également recueillis par Sébillot, en Bretagne, et je vais les comparer ${ }^{8} \gg$. Cette ouverture à d'autres auteurs amène Barbeau à lui suggérer des lectures et à lui présenter des auteurs. $\mathrm{Au}$ cours des années, Massicotte montre un intérêt constant pour les travaux de Paul Sébillot, d'Arthur Rossat, d'Henri Gaidoz et de Georges Doncieux. Plus tard, il s'intéresse à ceux de Prosper Convert et d'Arnold Van Gennep. Il consulte aussi entre autres les revues Traditions populaires (France), Folklore (Angleterre) et Journal of American Folklore (É.-U.). Marius Barbeau a dû quelquefois regretter son geste lorsque les deux n'étaient pas d'accord puisque la majorité des arguments de Massicotte était basée sur les méthodes de ces folkloristes réputés.

Le travail sur le terrain est des plus dynamiques. Au-delà de l'équipement technique, Marius Barbeau s'occupe aussi de faire relever les chants à la dactylo. Massicotte consacre ainsi une partie de ses soirées et de ses vacances aux cueillettes de folklore. Pour sa part, Barbeau partage ses terrains entre la côte Ouest et le Québec. Dans leur correspondance, ils se motivent l'un et l'autre en mentionnant l'importance de leurs cueillettes respectives, en partagent leurs découvertes et leur enthousiasme.

\section{Divergences sur les enjeux de la diffusion}

Dès l'automne 1917, Marius Barbeau lui demande de revoir un manuscrit. Des divergences d'opinions apparaissent rapidement sur les publics ciblés, la manière de présenter l'information et les objectifs de la publication. Massicotte lui écrit :

Par les journaux et les revues, vous atteignez des profanes qui ignorent tout ou presque tout du travail que coûtent les récoltes de folkloristes; des profanes qui ne savent rien ou presque des diverses espèces de chansons qui se conservent chez le peuple. Or, comme la publication de ces pièces est surtout faite - si je ne m'abuse - dans le but d'amorcer le public, ne vaut-il pas mieux rendre l'appât attrayant de toute façon ? Le détail historique, anecdotique, etc. ne nuirait pas. ${ }^{9}$

Pour atteindre ce but, il lui suggère de donner la provenance de la chanson, l'origine du chanteur, son âge et sa profession accompagné d'un contexte historique du chant. Plus tard, en 1918, Barbeau lui demande de commenter une étude d'Arthur Rossat sur les chants. Selon Massicotte : «L'ouvrage de Rossat est admirable. C'est bien comme cela que devrait être publié notre

8. Afeul, Fonds Édouard-Zotique Massicotte (F837), É-Z. Massicotte à M. Barbeau, 4 juillet 1917.

9. Ibid., 12 novembre 1917. 
collection ou qui aurait dû : les mêmes genres ensemble et les variantes à la queue leu leu ${ }^{10} \gg$. Ils ont plusieurs échanges sur ce sujet et, pour convaincre l'anthropologue, Massicotte applique sa suggestion à ses chansons. On devine la réaction de Marius Barbeau dans une autre missive de l'archiviste :

Comme vous n'êtes pas tendre pour les auteurs naïfs qui mettent un résumé de la chanson au début. [...] Mais ne pourrait-on pas indiquer le thème sec. Je ne tiens pas à cette idée outre mesure. Je ne cherche qu'à rendre ces machines aussi agréables ou attrayantes que possible. Il me semble avoir vu cette méthode employée quelque part. Me suis-je trompé ? Rossat ne met-il rien au début ? Donne-t-il ses chansons nues comme le discours d'un académicien ? ${ }^{11}$

Dans l'ensemble, malgré leurs divergences, Marius Barbeau apprécie les commentaires, intègre quelques suggestions et leur collaboration demeure dynamique. Mais, tout ceci change avec le projet des veillées de folklore.

En mai 1918, Marius Barbeau donne une conférence devant les membres de la Société historique de Montréal. Sa causerie frappe les esprits puisque quelques jours plus tard, Massicotte l'informe d'un projet de veillée de folklore publique prévue à l'automne ${ }^{12}$. Il lui demande aussi s'il veut y collaborer. Barbeau accepte. En septembre, une équipe de travail s'organise à Montréal. Elle ébauche un programme et conçoit une scénographie. Cependant, malgré plusieurs rappels, elle demeure sans nouvelle de l'anthropologue. Ce dernier explique à Massicotte qu'il manque d'enthousiasme parce qu'il ne croit pas au succès commercial de la veillée ${ }^{13}$. À cette époque, les recherches sur le folklore ne sont pas subventionnées. Cependant, elles sont essentielles pour les publications et pour intéresser les érudits canadiens-français. Le caractère novateur des veillées publiques constitue un risque financier pour la Société historique. Par ailleurs, ces soirées confrontent aussi les convictions de Marius Barbeau envers le folklore. Si elles s'avéraient un échec, cela pourrait compromettre son projet d'étudier le folklore canadien-français ${ }^{14}$. À l'automne, il est toujours muet, ce qui oblige les organisateurs à reporter l'activité au printemps 1919. Par la suite, Barbeau développe un nouvel intérêt pour les soirées. Toutefois, d'autres différends se manifestent. Ainsi, après avoir revu le programme préliminaire, il recommande d'ajouter une projection lumineuse d'objets du terroir. Massicotte n'est pas d'accord, car il trouve la soirée déjà trop longue. Ce dernier veut aussi que les chanteurs soient accompagnés, mais Barbeau suggère le contraire. Il écrit : « comme les

10. Ibid., 9 février 1918.

11. Ibid., 23 février 1918.

12. Ibid., 2 juin 1918. La conférence fut donnée le 29 mai.

13. Centre des archives de Montréal, Fonds Édouard-Zotique Massicotte (SHM12), M. Barbeau à É.-Z. Massicotte, 14 juillet 1918.

14. Tous les fonds d'archives mentionnent des oppositions aux recherches sur le folklore que l'on juge passéistes et les débuts laborieux de la $2^{\mathrm{e}}$ section locale de la Société américaine de folklore. 
auditeurs ont des goûts aristocratiques, ce n'est pas une bonne idée d'utiliser des artistes populaires sur la scène - [ce] serait fatigant et déconcertant pour eux d'affronter le public sans répit ${ }^{15} »$. Des conflits surgissent aussi sur le choix des artistes et les délais trop longs à répondre. Dans l'ensemble, Victor Morin concilie les points de vue et les humeurs de chacun.

Les soirées suscitent un engouement sans précédent et les organisateurs se retrouvent avec une surréservation de sièges les obligeant à offrir une deuxième veillée. Enthousiaste, Barbeau suggère de présenter non pas une, mais deux autres soirées. Il propose aussi d'engager l'interprète américaine Lorraine Wyman pour les prochaines séances. Pour sa part, Massicotte s'oppose à l'idée de deux soirées, car il craint la surexposition du folklore et leur commercialisation par d'autres. Il n'est pas d'accord non plus avec la venue de Wyman qui, malgré son grand talent, demeure une anglophone interprétant des chants en français ${ }^{16}$. Barbeau insiste, s'acharne même dans ses nombreuses missives au point où l'archiviste perd patience :

Vous tournez au mercantilisme par amour de l'art en voulant la future soirée parce qu'elle procurera des fonds au folk[lore]. Ne soyez pas ingrat. Cette soirée, dont la deuxième n'est que le prolongement, marque une époque dans l'histoire $\mathrm{du}$ folklore canadien-français sur laquelle on pourra s'appuyer. ${ }^{17}$

Grâce au succès commercial des veillées, la Société historique de Montréal et la section locale de la Société de folklore américaine se décident de publier un compte rendu. Édouard-Zotique Massicotte supervise la production et commente les textes. Cependant, l'édition des Veillées du bon vieux temps amène d'autres disputes notamment à cause de l'attitude belliqueuse de Barbeau qui met au jour ses profondes frustrations. D'après leurs échanges, le folklore et Marius Barbeau auraient fait l'objet de plusieurs critiques acerbes et ce dernier souhaite riposter dans l'avant-propos. À l'automne 1919, Massicotte lui fait une mise en garde : «Au sujet de la préface : croyez-moi, le linge sale de famille doit se laver en famille. Il ne faut pas signaler à l'attention publique que nos éminences artistiques et littéraires se querellent comme des cochers de fiacre ou des porte-à-faux ${ }^{18} »$. À la suite de plusieurs autres échanges acrimonieux, toujours sur le même sujet, Massicotte finit par le menacer d'abandonner le projet :

Je reviens à bouquins jaunis. De tout temps, les bouquins jaunis ont été considérés la marotte des collectionneurs, des chercheurs, des amateurs de choses

15. Centre des archives de l'Université de Montréal, Fonds Victor Morin (P56), M. Barbeau à V. Morin, 11 mars 1919.

16. Centre des archives de Montréal, Fonds Édouard-Zotique Massicotte (SHM12), É.-Z. Massicotte à M. Barbeau, 4 février 1929.

17. Afeul, Fonds Édouard-Zotique Massicotte (F837), É-Z. Massicotte à M. Barbeau, 8 avril 1919.

18. Ibid., 18 octobre 1919. 
d'autrefois. [...] Je ne connais pas d'amateurs de bouquins jaunis qui n'ont pas en eux l'étoffe d'un folkloriste. [...] La vie sociale n'est possible qu'à force de concession. L'amitié doit aller plus loin. Si vous tenez absolument à salir vos ennemis, au moins n'éclaboussez pas vos amis par la même occasion. Je maintiens que bouquins jaunis m’offense et si vous le laissez, je dételle ! Prenez garde jeune homme, votre agressivité prend des proportions. Cela vous jouera des tours. ${ }^{19}$

Un malaise s'installe entre eux et ils espacent leur correspondance. L'enthousiasme de Massicotte pour le folklore persiste tout comme l'aide technique de Marius Barbeau. Mais d'autres conflits surgissent, entre autres, à cause de la lenteur de l'anthropologue à retourner les textes de chansons en format dactylographié et à propos de droits d'auteurs. La correspondance indique qu'il y a une entente, ou, à tout le moins, l'idée de tirer profit des collectes lors de publications. Sur ce propos, Massicotte est pointilleux, intraitable même. Il s'assure que ses contributions sont bien indiquées lorsque Barbeau utilise ses données de terrain ; il le questionne de temps à autre sur le partage des profits. En 1920, une tentative d'usurpation de chansons données à Massicotte donne lieu à d'autres échanges rudes :

Vous avez raison lorsque vous dites que certaines personnes sont plus à l'aise quand elles travaillent sous une tutelle éloignée et qui ne se fait pas trop sentir. Vous avez également raison, mais un peu moins, c'est-à-dire d'une autre façon, quand vous dites que l'oiseau est devenu bilieux et rempli d'amertume. Certes, je reconnais que l'oiseau est très sensible. D'autres parts, Marius de 1919-20 est-il bien le Marius de 1917-18 ? Ne lui arrive-t-il pas, par-ci par-là, de brusquer ses amis, d'échapper des mots qui font l'effet d'une taloche ; de décider sans consulter les intéressés ?

Il n'était pas ainsi quand je l'ai connu. C'est par des [paroles] fleuries qu'il conduisait ses adeptes dans le domaine du folklore ! Nul n'avait plus d'indulgence, plus de diplomatie que maître. Possible que le changement se soit produit hors de sa connaissance. Suis-je le seul à le remarquer $?^{20}$

Toutefois, l'incident le plus important survient à l'automne lorsque Marius Barbeau l'avise qu'il ne sera pas rémunéré pour ses travaux d'été :

Je croyais vous avoir donné les indications nécessaires sur les procédures à suivre ; peut-être aviez-vous oublié ? Vous n'étiez pas pour mettre de salaire pour vous-même sur le compte, mais seulement pour vos informateurs. [...] Je regrette fort ce contretemps [...] parce que j'espérais établir par là, avec votre aide, un précédent pour vous et d'autres collègues. ${ }^{21}$

19. Ibid., [novembre 1919].

20. Ibid., 3 février 1920.

21. Centre des archives de Montréal, Fonds Édouard-Zotique Massicotte (SHM12), M. Barbeau à É.-Z. Massicotte, 11 octobre 1920. 
L'argent est un point sensible chez Édouard-Zotique Massicotte. Sa correspondance mentionne à plusieurs reprises des charges financières importantes avec des moyens plutôt limités ${ }^{22}$. On le devine, il réplique rapidement. Énervé, l'archiviste écrit :

Je ne devrais pas demander de salaire pour moi ??? J'ai pourtant bien suivi le modèle que vous m'avez envoyé \& où le premier item est : wages for 25 days @ $\$ 3.00$ a day $=\$ 75$. J'écris à ce jour à $m$. Sapir : Dear Sir, $m$. Barbeau vient de m'apprendre que je suis un «profiter », un exploiteur, que je n'ai droit à aucun salaire pour mon travail cet été. [...] Je n'ai jamais compris que j'allais faire une enquête, au loin, pour rien. Si j'avais compris, j'aurais travaillé à quelque chose qui m'aurait payée [sic]. J'en avais l'avantage et aussi le besoin. ${ }^{23}$

À la fin, Édouard-Zotique Massicotte sera rémunéré à même les fonds de la Société de folklore. Pour sa part, Marius Barbeau gagne son pari avec son employeur qui décide de réserver un budget dédié à des recherches sur le folklore au Québec. L'institution rembourse donc Massicotte qui se retrouve payé en trop!

Tout finit bien et tout semble réglé puisqu'Édouard-Zotique Massicotte envoie un devis pour ses travaux d'été qui est approuvé en 1921. Cependant, le cœur n'y est plus. En juillet, il avise Marius Barbeau qu'il préfère travailler sur des projets personnels. À l'automne, il suggère de faire dactylographier les chansons à Montréal grâce à l'argent non dépensé au cours de l'été. Massicotte évite ainsi d'autres disputes sur ce propos et reprend le contrôle de sa collection. Par la suite, il mettra surtout de l'ordre dans ses données.

L'année suivante, Marius Barbeau l'introduit aux croix de chemin et lui fournit le matériel technique pour les photographier. La période marque aussi la nomination de Massicotte à la Commission des monuments historiques à laquelle il consacrera toutes ses énergies. Il abandonne la cueillette de folklore pour se vouer à la photographie de biens culturels qui présentent un intérêt patrimonial selon la Commission. Il l'a convaincu également d'inclure dans sa liste des artéfacts associés à la tradition populaire tels que les croix de chemin et à la vie d'antan. Le musée d'Ottawa paie pour cette partie des travaux. La correspondance entre les deux folkloristes demeure laconique : liste des films à développer, échéanciers, factures, remboursements...

\section{Une relation renouvelée sur le respect mutuel}

À cette époque et pour deux autres années - jusqu'en 1924, Marius Barbeau

22. En 1913, après plusieurs repos forcés, Massicotte reçoit le diagnostic d'une insuffisance rénale chronique (maladie de Bright). Selon les périodes de l'année et la météo, il souffre de douleurs osseuses, de déprime, de migraines sévères, de problèmes nerveux et de concentration.

23. Afeul, Fonds Édouard-Zotique Massicotte (F837), É-Z. Massicotte à M. Barbeau, 20 octobre 1920. 
poursuit ses recherches sur la côte Ouest. L'éloignement entre les deux chercheurs se fait naturellement. Le hasard les amène à développer chacun une nouvelle passion et ils ressentent le besoin de la partager. En janvier 1925, Marius Barbeau reçoit cette missive :

Cher monsieur Barbeau, je viens de recevoir un ouvrage que vous devriez avoir dans votre collection. [...] Prosper Convert a réussi là-bas ce que nous avons fait en petit, avec les chansons de chantiers - représenter des scènes de la vie populaire par la chanson. Toutes ses représentations sont données par des gens du peuple : mais il faut voir le livre, le savourer, pour apercevoir tout ce que nous pourrions tirer de notre stock. ${ }^{24}$

Cet ouvrage est important, car il amène Édouard-Zotique Massicotte à inclure la thématique du folklore dans les défilés de la Société Saint-Jean-Baptiste de Montréal la même année. À l'époque, sa scénographie audacieuse favorise la patrimonialisation d'objets issus de la culture populaire, entre autres les chansons et les savoir-faire, qui correspondent aujourd'hui en partie au patrimoine immatériel inscrit dans la loi québécoise.

De son côté, Marius Barbeau découvre l'île d'Orléans. Il lui écrit :

Cher monsieur Massicotte, la tradition des beaux-arts établie par $\mathrm{M}^{\mathrm{gr}}$ de Laval est ma principale étude et je n'en ai jamais touché de plus captivante. J'étudie les églises, les plus intéressantes et anciennes, et leurs archives paroissiales qui fournissent les renseignements nécessaires sur leur origine et leur évolution. [...] J'étudie aussi la tradition artistique avec des survivants de l'ancienne école, particulièrement [Louis] Jobin, de Sainte-Anne. ${ }^{25}$

Cette découverte est également importante même si l'idée d'une tradition artistique en Nouvelle-France est une erreur historique. Ce concept, que Marius Barbeau mettra de l'avant, donne l'élan nécessaire à l'émergence d'une histoire de l'art canadien.

Par la suite, leurs échanges sont plus cordiaux et la relation est égalitaire. En 1927, Marius Barbeau sollicite son aide lors d'un festival de folklore à

24. Ibid., 25 janvier 1925.

25. Centre des archives de Montréal, Fonds Édouard-Zotique Massicotte (SHM12), M. Barbeau à É.-Z. Massicotte, 19 août 1925. Plusieurs historiens de l'art ont souscrit à cette idée de Marius Barbeau dont Gérard Morisset, Jean Palardy, les anglophones Russel Harper, Robert Hubbard et Graham McInness. En 1975, l'historien Peter N. Moogk présente une étude exhaustive où il conclut qu'une telle école n'a jamais existé ou du moins qu'il n'en subsiste aucune preuve suffisante. Le modèle regroupant des apprentis serait un phénomène inusité pour une époque où la transmission du savoir artisanal en France se faisait auprès des artisans eux-mêmes, dans un système de maître-apprenti. La fabrication de Barbeau serait un anachronisme basé sur des réalités du XIX ${ }^{\mathrm{e}}$ siècle. L'historien de l'art François-Marc Gagnon a revu l'étude de Moogk et validé sa position (Robert Derome et Madeleine Landry, L'Art sacré en Amérique française : le trésor de la Côte-de-Beaupré, Sillery, Québec, Éditions du Septentrion ; Nouveau monde, 2005, p. 51.) Publiée en 1920 (Montréal, G. Ducharme), l'étude Une maîtrise d'art en Canada, 1800-1823, d'Émile Vaillancourt, est connue de Barbeau. Elle retrace le quotidien de l'école d'art du sculpteur Louis Quévillon. Il est fort probable qu'elle ait modelé le propos de l'anthropologue. 
Québec organisé par le Canadien Pacifique. Ils se consultent sur le choix des chanteurs, des chansons et de certaines activités. Malgré son enthousiasme, et une invitation au festival toutes dépenses payées, Massicotte décide de rester à Montréal. Marius Barbeau persévère. Rusé, il profite de l'intérêt de l'archiviste pour les artistes et de son penchant pour l'exactitude historique lorsqu'il lui écrit : «J'ai fait dix à douze jours de recherches très actives au sujet de [Cornélius] Krieghoff à Québec et à Montréal et j'ai trouvé beaucoup de choses. Je suis maintenant bien renseigné et je vais écrire avec enthousiasme à son sujet. [...] Il est évident que Krieghoff ne s'est jamais marié légalement ${ }^{26} »$. La réponse, teintée par la rigueur méthodique d'ÉdouardZotique Massicotte, vient rapidement : «Allez-y prudemment sur le mariage de Krieghoff. Il peut avoir eu lieu légalement dans un district où l'on n'a pas un index général comme à Montréa $\left.\right|^{27} \gg$.

Cet échange est exemplaire. Pendant encore plusieurs années, Marius Barbeau demandera à Massicotte de commenter ses manuscrits. Les opinions de l'archiviste demeurent sobres et fidèles à ses idées sur les publics et l'importance de vulgariser l'information. Des notes de remerciement de l'anthropologue signalent son appréciation et l'intégration de plusieurs suggestions. Pour sa part, Massicotte profite également des connaissances de Marius Barbeau et recherche son opinion lorsqu'il conçoit les thèmes des défilés de la Société Saint-Jean-Baptiste à Montréal notamment sur les chansons, les contes et les légendes.

En 1935, Marius Barbeau relance encore Édouard-Zotique Massicotte pour une collaboration de recherche et d'écriture. Et ce dernier refuse à nouveau :

J'ai bien des notes, j'ai bien des idées, malheureusement, j'ai à répondre à une correspondance de plus en plus volumineuse relative à des questions historiques et généalogiques. [...] Pendant ce temps, je vieillis et mes notes dorment, il vous incombera donc la tâche amicale de laisser voir que je vous fus un collabo[rateur] aussi dévoué et tenace que désintéressé. ${ }^{28}$

Au-delà de ce second refus, l'estime d'Édouard-Zotique Massicotte envers Marius Barbeau est sincère. Ce dernier fait partie de la liste restreinte des invités de Massicotte lors de la remise d'un doctorat honorifique de l'Université de Montréal. Massicotte se fait aussi un point d'honneur à lire les publica-

26. Centre des archives de Montréal, Fonds Édouard-Zotique Massicotte (SHM12), M. Barbeau à É.-Z. Massicotte, 30 octobre 1933.

27. Id., É.-Z. Massicotte à M. Barbeau, 6 novembre 1933. Cornelius Krieghoff s'est marié une première fois à Amsterdam, puis à Manhattan dans l'état de New York (Raymond Vézina, « Krieghoff, Cornelius », Dictionnaire biographique du Canada, vol. 10, Université Laval/University of Toronto, 2003-, www.biographi.ca/fr/bio/krieghoff_cornelius_10F.html, consulté le 21 janvier 2015).

28. Afeul, Fonds Édouard-Zotique Massicotte (F837), É-Z. Massicotte à M. Barbeau 14 mai 1935. 
tions de Barbeau et à lui envoyer un mot d'encouragement à poursuivre ses recherches. Pour sa part, Marius Barbeau témoigne aussi de la sollicitude pour l'archiviste. Il lui envoie systématiquement tous les ouvrages qu'il publie. Si Massicotte mentionne une étude qui l'intéresse, il s'empresse de la faire acheter par le musée et de la lui prêter. Lorsque Massicotte est déprimé, et cela arrive assez souvent, il lui écrit un mot d'encouragement :

Vers la fin de votre dernière lettre, vous semblez regretter un peu d'avoir recueilli tant de chansons populaires dans le passé au lieu d'écrire un ou deux volumes sur la ville de Montréal. Il ne faudrait pas que ces regrets soient trop graves, car je suis sûr qu'avec le temps et la publication graduelle de ces chansons, on aura pour vous une grande reconnaissance à l'endroit de votre cueillette. Les chansons et leurs mélodies sont d'un intérêt universel; elles gagneront avec le temps encore plus de faveurs. ${ }^{29}$

Leur dernier échange s'est produit en juin 1947, quelques mois avant le décès d'Édouard-Zotique Massicotte. Les deux chercheurs y discutaient de corrections à apporter dans une étude de Marius Barbeau.

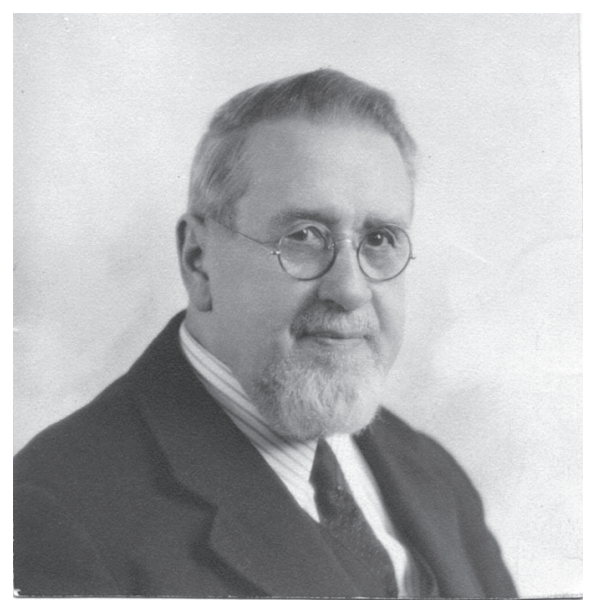

Édouard-Zotique Massicotte, vers 1938

Photographe inconnu

Source : Reproduit avec l'autorisation de Bibliothèque et Archives nationales du Québec

Collection Centre d'archives de Québec

(P1000, S4, D83, PM52-1)

29. Centre des archives de Montréal, Fonds Édouard-Zotique Massicotte (SHM12), M. Barbeau à É.-Z. Massicotte, 21 mars 1939. 